\title{
A computer model of drafting effects on collective behavior in elite $10,000 \mathrm{~m}$ runners
}

\begin{tabular}{|r|l|}
\hline Journal: & International Journal of Sports Physiology and Performance \\
\hline Manuscript ID & IJSPP.2016-0026.R1 \\
\hline Manuscript Type: & Original Investigation \\
\hline Keywords: & Pacing, Endurance, Running, Modelling \\
\hline \multicolumn{2}{l}{} \\
\hline
\end{tabular}


1 2

3

4

5

Title: A computer model of drafting effects on collective behavior in elite 10,000 m runners

Submission Type: Original Investigation

Authors and Affiliations: Hugh Trenchard ${ }^{1}$, Andrew Renfree ${ }^{2}$, Derek M. Peters ${ }^{2,3}$

${ }^{1} 805647$ Michigan Street, Victoria, British Columbia, Canada V8V 1S9

Tel: +1 2504720718

${ }^{2}$ Institute of Sport \& Exercise Science, University of Worcester, Henwick Grove, Worcester, United Kingdom, WR2 6AJ

${ }^{3}$ Faculty of Health \& Sport Sciences, University of Agder, Kristiansand, Norway

Corresponding Author: Andrew Renfree, Institute of Sport \& Exercise Science, University of Worcester, Henwick Grove, Worcester, United Kingdom, WR2 6AJ

Tel: $+44(0) 1905855376$

Fax: +44 (0)1905 855132

Email: a.renfree@worc.ac.uk

Preferred Running Head: Modelling collective behavior in 10,000 m running

Abstract Word Count: 246

Text-Only Word Count: 3576

Number of Figures: 5 


\section{$47 \quad$ Abstract}

\section{Purpose}

72

77

78

79

80

Drafting in cycling influences collective behaviour of pelotons. Whilst evidence for collective behaviour in competitive running events exists, it is not clear if this results from energetic savings conferred by drafting. This study modelled the effects of drafting on behavior in elite 10,000 m runners.

\section{Methods}

Using performance data from a men's elite 10,000 m track running event, computer simulations were constructed using Netlogo 5.1 to test the effects of three different drafting quantities on collective behaviour: no drafting, drafting to $3 \mathrm{~m}$ behind with up to $\sim 8 \%$ energy savings (a realistic running draft); and drafting up to $3 \mathrm{~m}$ behind with up to $38 \%$ energy savings (a realistic cycling draft). Three measures of collective behaviour were analysed in each condition; mean speed, mean group stretch (distance between first and last placed runner), and Runner Convergence Ratio (RCR) which represents the degree of drafting benefit obtained by the follower in a pair of coupled runners.

\section{Results}

Mean speeds were $6.32 \pm 0.28 \mathrm{~m} . \mathrm{s}^{-1}, 5.57 \pm 0.18 \mathrm{~m} . \mathrm{s}^{-1}$, and $5.51 \pm 0.13 \mathrm{~m} . \mathrm{s}^{-1}$ in the cycling draft, runner draft, and no draft conditions respectively (all $P<0.001$ ). RCR was lower in the cycling draft condition, but did not differ between the other two. Mean stretch did not differ between conditions.

\section{Conclusions}

Collective behaviours observed in running events cannot be fully explained through energetic savings conferred by realistic drafting benefits. They may therefore result from other, possibly psychological, processes. The benefits or otherwise of engaging in such behavior are, as yet, unclear.

\section{Keywords}

Pacing, Endurance, Running, Modelling 


\section{Introduction}

Research has explored the mechanisms through which 'pacing', which reflects the strategy for expending effort during athletic contests ${ }^{1}$, is regulated. Whilst much of this work has focussed on internal regulatory processes, including the role of the momentary Rating of Perceived Exertion $(\mathrm{RPE})^{2}$, the Hazard Score ${ }^{3}$, and emotion ${ }^{4,5}$, two recent reviews ${ }^{6,7}$ have suggested that regulation is achieved through a continual process of decision-making. A key feature of these decisionmaking processes is that choices are made based on interpretation of data of either internal or external origin, which are 'perceived' to require a particular decision to be made and a course of action taken at that moment in time. Indeed, Smits et al. ${ }^{7}$ have identified that in order to explain athletic pacing decisions it may well be necessary to adopt an ecological approach that enhances understanding of how perception and action are coupled in determining behavior. Given that athletes often compete in direct proximity to one another without separation due to individual lane allocations, it is interesting that relatively few authors have explored the nature of interactions between competitors in endurance athletic events, and their influence on pacing behaviors.

Different pacing strategies have been shown in elite female marathon runners resulting in athletes achieving different absolute performance levels ${ }^{8}$, whereby slower athletes adopted similar starting speeds to the faster athletes who finished in the leading positions. These overly ambitious starting speeds resulted in progressive deceleration throughout the race, and overall race pacing profiles characterized by a 'positive split', whereby the second half of the race was run more slowly than the first. Although similar findings are evident in elite male athletes at the World Cross Country Championships ${ }^{9,10}$, it is not clear why runners of differing performance ability tend to adopt similar starting speeds. It may be evidence for a human tendency towards collective behavior influencing pacing decisions, as in complex decision-making environments the easiest decision is simply to do the same as everybody else ${ }^{11}$, which may explain behaviors in other human environments including pedestrian interactions ${ }^{12}$ and market trading ${ }^{13}$. Although the precise mechanisms underlying these behaviors are not fully understood, such complex biological systems may well result from individual agents following simple rules governing the nature of their interactions with others ${ }^{14}$.

Among pelotons evidence indicates collective behavior self-organizes from cyclists' local interactions. Pelotons are groups of cyclists coupled by the energy-savings of drafting ${ }^{15}$, and may include as many as 200 individuals. Trenchard ${ }^{15}$ found that pelotons exhibit protocooperative behavior, which emerges as a function of cyclists'capacity to share the most costly front positions where aerodynamic drag is highest. As speeds vary, three main collective conditions emerge: when speeds are low relative to the cyclists' maximal sustainable outputs (MSO), individuals naturally cooperate by sharing the metabolically more costly front positions. In this condition pelotons are compact and roughly circular in shape. As speeds increase eventually the protocooperative threshold is reached whereby weaker cyclists are unable to share the costly front-positions, and must maintain drafting positions to sustain the speed of the leading riders. In this condition pelotons are single-file formation, and highly stretched. At yet higher speeds, when weaker cyclists are unable to keep up with stronger cyclists even by drafting, a second threshold is reached as cyclists decouple and form smaller sub-groups. Both protocooperative and decoupling thresholds depend on the differentials between MSOs of the weaker and stronger 
riders, and the drafting quantity (which may be zero). Therefore, higher drafting quantities permit greater MSO differential before either threshold is reached. A key prediction of protocooperative behavior is that groups tend to sort so that the MSO variation range among the group of cyclists approximately corresponds to the energy savings of drafting.

In running events the energetic savings from drafting are smaller due to the lower speeds achieved $^{16-18}$, and the nature of any resulting protocooperative behavior is therefore largely unknown. Whilst Hanley ${ }^{19}$ has demonstrated that competitors in the World Half Marathon championships often form groups, and those athletes who run in groups throughout tend to display a greater 'endspurt' in the final stages, the reasons for this are unclear. It is plausible that this could result from the athletes achieving speeds whereby there is some energetic benefit from drafting behavior ${ }^{20}$, or because of a reduced cognitive load due to a reduction in the need to make continuous pacing decisions ${ }^{21}$, or some combination of the two.

Our aim therefore was to model collective behavior of a group of elite distance runners during competition in order to determine the degree to which collective behavior may be influenced by energetic savings incurred through drafting. We hypothesized that models would suggest drafting benefits will influence collective behavior during a $10,000 \mathrm{~m}$ running race.

\section{Method}

A quasi-experimental design was used to address the aim of the study which had received prior ethical approval from the University of Worcester. Final results and official split times (individual 100m segments) of all starters ( $\mathrm{n}=32$ ) in the Men's 10,000 m event at the 2013 IAAF World Championships were accessed via the championship website (http://media.aws.iaaf.org/competitiondocuments/pdf/4873/AT-10K-M-f--1--.RS7.pdf?v=1733122098 ) along with seasons best (SB) performances for all competitors. This event was selected because of the relatively homogenous performance characteristics of the competitors, and the high frequency of timing data available.

To analyze collective running dynamics, we adapted the modified ${ }^{15}$ peloton simulation originally developed by Trenchard et al. ${ }^{22}$ This model incorporates maximal sustainable output (MSO) thresholds whereby cyclists decelerate when MSOs are exceeded relative to a pacesetter; and build upon Ratemero's peloton model ${ }^{23}$ and flocking dynamics whereby group mean $x$ and $y$ coordinate positions generate cohesion and separation parameters ${ }^{23}$. Simulations were performed using Netlogo 5.1, a multi-agent computer modelling platform ${ }^{24}$. The adapted runner model involved simple modifications to the peloton threshold equations ${ }^{22}$, as follows:

$$
R C R={\underline{S_{\text {front }}}}_{\mathrm{MSO}_{\text {follow }}}^{\mathrm{d}}
$$

Where "RCR" is the "runner convergence ratio", describing two coupled runners whereby the leader sets the pace and the follower may obtain a drafting benefit. If there is drafting quantity, RCR reduces accordingly, and if there is no drafting quantity, RCR is simply a ratio of the pacesetter's speed to the follower's MSO; 
"S $\mathrm{S}_{\text {front }}$ " is the front runner's speed, "MSO follow" is the follower's MSO in terms of speed (m/s) (for the purposes of this study we utilised the athletes SB times as representing MSO); and " $d$ " is the drafting coefficient obtained from:

$$
d=0.62-0.0104 d_{w}+0.0104 d_{w}+0.0452 d_{w}^{2}
$$

Where $d_{w}$ is distance between rear wheel of front rider, and front wheel of drafting rider in meters.

Equation (2) was developed by $\mathrm{Olds}^{25}$ using Kyle's published data ${ }^{16}$, which indicated energy savings of up to approximately $38 \%$ in cyclists, depending on wheel spacing. Whilst this equation does not reflect realistic drafting advantage for runners, we used it here as one of three drafting quantities to test the effects of drafting on collective running dynamics. If wheel spacing is $3 \mathrm{~m}$ or greater, $d$ is assumed to be 1 (no drafting benefit) ${ }^{26}$.

For runners, since the speeds are considerably slower than in cycling, the drafting benefit (1- $d$ ) is smaller. Kyle ${ }^{16}$ found a $4 \%$ reduction in $\mathrm{VO}_{2}$ at $6 \mathrm{~m}^{-1} \mathrm{~s}^{-1}$ when drafting at $1 \mathrm{~m}$; $\mathrm{Pugh}^{17}$ found a $6.5 \%$ reduction at $4.5 \mathrm{~m} . \mathrm{s}^{-1}$ with a wind velocity of $6 \mathrm{~m} . \mathrm{s}^{-1}$ when drafting at $1 \mathrm{~m}$. Similarly, Davies $^{19}$ found $4 \%$ reduction at $6 \mathrm{~m} \cdot \mathrm{s}^{-1}$ and $2 \%$ at $5 \mathrm{~m} \cdot \mathrm{s}^{-1}$.

Further, applying empirical drafting quantities again reported in cycling by McCole et al. ${ }^{26}$ we derived the following regression equation:

$$
d^{\prime}=-0.036 * \mathrm{~s}_{\text {front }}+1.14
$$

Where " $\mathrm{S}_{\text {front }}$ " is the speed in $\mathrm{m} . \mathrm{s}^{-1}, d$ ' is approximately $0.92(8 \%$ reduction in metabolic requirement), which is consistent with both the high end of the range of empirical findings noted, and the actual mean speed of the runners in the Moscow 10,000 m $\left(5.98 \mathrm{~m} \cdot \mathrm{s}^{-1}\right)$.

Equation (3) is similar to (2) except $d$ ' is constant (0.92), whilst $d$ varies according to distance up to $3 \mathrm{~m}^{*}$. The empirical data ${ }^{16-18}$ does not clearly indicate whether drafting abruptly drops to zero at $1 \mathrm{~m}$ for runners, or whether it tails off up to $3 \mathrm{~m}$, as the evidence indicates for cyclists. Here we err on the side of greater drafting benefit for runners to obtain clearer evidence of any effect that drafting might have on collective running behavior. We infer negligible drafting benefit at angles, but allow a 15 degree "comet's tail" 26 drafting effect to runners' sides, and zero at greater angles.

Further, to obtain the drafting quantities for runners whereby drafting benefit decreases with distance between runners, we applied the equation:

$$
d=0.92-2.667 \times 10^{-3} d w+3.667 \times 10^{-3} d w^{3}
$$

Thus if RCR $>1$ for two runners, the follower cannot sustain the speed set by the leader and must decelerate to a speed less than or equal to the speed equivalent to that runner's MSO, as shown in the following equations, as adapted from Trenchard et al. ${ }^{22}$ : 
First obtain the front runner's speed in excess of $\mathrm{RCR}=1$ :

$$
\text { Speed }_{e}=\frac{(M S O * R C R)}{d}
$$

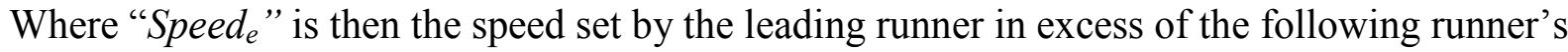
possible speed at MSO.

Then obtain the speed for the following runner at MSO:

$$
\text { Speed }_{t d}=M S O / d
$$

Where "Speed $t d$ " is a runner's speed at his MSO, given the possible increase in speed facilitated by the drafting benefit (if any). To obtain a runner's required speed reduction in order to resume running at MSO, find the difference between Speed $_{e}$ and Speed $_{t d}$ :

$$
\text { Speed }_{r}=\text { Speed }_{e}-\text { Speed }_{t d}
$$

If a runner incurs additional metabolic disruption as a result of the speed exceeding the metabolic cost of running at their MSO, fatigue would be expected to induce decelerations to a speed below his MSO, and not to a speed equivalent to MSO. To model this, we applied an additional random deceleration factor:

$$
\text { Speed }_{r}=\text { Speed }_{e}-\text { Speed }_{t d}+\Delta \mathrm{s}
$$

Where "Speed' $r$ " is the final speed due to deceleration, where $\Delta$ is the noted small positive random individual deceleration quantity. A relatively small random acceleration was generated by adding a random quantity to the cohesion parameter noted earlier.

With these model adaptations, to test the effect of drafting on runners' collective dynamics, we conducted 30 simulation trials for each of three experimental drafting quantities:

1. No drafting benefit ("no draft condition").

2. Drafting benefit up to $3 \mathrm{~m}$ behind other runners within a 15 degree cone centred around the current heading of the runner ahead, using equation (3) ("runner draft condition").

3. Drafting benefit up to $3 \mathrm{~m}$ behind other runners within a 15 degree cone, centred around the current heading of the runner ahead using equation (2) ("cyclist draft condition").

Simulation duration was 27:21.6 (1642 s) the fastest finishing time in the race. Accumulated times for runners who were first at each $100 \mathrm{~m}$ were used as pacesetter splits for each $100 \mathrm{~m}$ interval, converted to speeds $\left(\mathrm{m}_{\mathrm{s}} \mathrm{s}^{-1}\right)$, as shown in Figure 1.

\footnotetext{
**Insert Figure 1 near here**
} 
Thus there were 100 pacesetter speeds during each of the simulation races, with these speeds taken as stable during each intervening $100 \mathrm{~m}$. Across the 90 simulated trials, runners constantly adjusted their speeds, distances and positions relative to pacesetter speeds and varying draft conditions, according to equations (5-8).

\section{**insert Figure 2 near here**}

Unknown was the effect of drafting quantities on runners' RCRs, speeds, and stretch. The RCR indicates whether there is any available energetic resources that would allow for accelerations (i.e. if $\mathrm{RCR}<1$, runners have metabolic "room" to accelerate). Stretch is the distance (m) between the front runner and the last runner; in the simulation stretch equals the maximum $\mathrm{x}$-coordinate minus the minimum x-coordinate in which an agent appears, scaled to meters, a value that changes constantly. To analyze the data, we used Excel 97-2003 and NCSS 2007 for descriptive statistics and ANOVA. Statistical significance was accepted at $\mathrm{P}<0.01$ due to the comparatively large sample of data from 30 simulation trials for each variable where each simulation second (1642 s per simulation) represents a data point, yielding 49,260 data points for each of nine variables (RCR, stretch, speed; multiplied by: no draft, runner draft, and cyclist draft). Effect size was calculated using Cohen's $d^{27}$ as an additional statistical metric. We apply Cohen's classified effect sizes small $(d=0.2)$, medium $(d=0.5)$, and large $(d \geq 0.8){ }^{27}$

\section{Results}

The mean speed maintained in the cyclist draft condition $\left(6.32 \pm 0.28 \mathrm{~m} . \mathrm{s}^{-1}, 99 \% \mathrm{CI}=6.317,-\right.$ 6.323) was higher than in the no $\operatorname{draft}(P<0.001, d=2.907)$ and runner $\operatorname{draft}(P=<0.001, d=$ $2.686)$ conditions. Speed also differed between the no draft $\left(5.51 \pm 0.13 \mathrm{~m} \cdot \mathrm{s}^{-1}, 99 \% \mathrm{CI}=5.506\right.$, $5.509)$ and runner draft conditions $\left(5.57 \pm 0.18 \mathrm{~m} . \mathrm{s}^{-1}, 99 \% \mathrm{CI}=5.568,5.572\right)(P<0.001, d=$ 0.3553 ) (Figure 3 ), where there is low to medium effect, but effect overall is very low relative to the effects of the draft condition on speed.

\section{**Insert Figure 3 near here**}

The RCR was lower in the cyclist draft condition $(0.88 \pm 0.06,99 \% \mathrm{CI}=0.8822,0.8835)$ than in the no draft $(P<0.001, d=2.0989)$ and runner $\operatorname{draft}(P<0.001, d=2.0512)$ conditions, and large effect. There were no differences, and small effect, found between the no draft $(1.00 \pm 0.04,99 \%$ $\mathrm{CI}=1.0011,1.0021)$ and runner draft conditions $(1.00 \pm 0.04,99 \% \mathrm{CI}=0.9984,0.9993)(P=$ $0.1098 ; d=0.0668$ ) (Figure 4).

\section{**Insert Figure 4 near here**}

There were no differences in mean stretch between any of the drafting conditions (Figure 5), whereby in the cyclist draft condition it was $158.71 \pm 113.28 \mathrm{~m}(99 \% \mathrm{CI}=157.39,160.02)$, in the no draft condition it was $125.42 \pm 68.81 \mathrm{~m}(99 \% \mathrm{CI}=124.62,126.22)$, and the runner draft condition it was $146.99 \pm 85.89 \mathrm{~m}(99 \% \mathrm{CI}=145.99,147.99)$ 


\section{**Insert Figure 5 near here** Discussion}

Our study sought to model the impact of three different drafting conditions on athlete performance and collective behavior in 90 computer simulated running races using the original data from the Men's 10,000 m event at the 2013 IAAF World Championships. We hypothesized that the potential energetic benefits resulting from effective drafting would be more apparent in the cycling draft condition compared with the running draft condition and that both would be better than the no draft condition.

The mean speed and RCR results (Figures $3 \& 4$ ) demonstrated a similar pattern in that runners were able to maintain greater mean speed and lower RCR in the cyclist draft condition compared with both other conditions. There was no difference in RCR between the runner and no draft conditions. Although the difference in speed between the no drafting and runner drafting conditions achieved our threshold for accepting statistical significance, it should be noted that the effect size was much smaller than between the other conditions. Our results suggest that the previously documented energetic benefits achievable through drafting in cycling studies are unlikely to be realised in running events. In the more realistic simulated running condition, there were very small performance benefits realised in terms of mean speed, and RCR did not differ from the no draft condition. No differences were found in mean stretch between any of the draft conditions (Figure 5) indicating that the overall spread of the field of athletes (from first to last position) was not influenced by either the speed of the race or the RCR.

Since our results indicate no significant effect of drafting on collective dynamics, there is no evidence that drafting has any bearing on the finding that acceleration capacity near the end of a race is greater in athletes who have run as part of a group throughout ${ }^{19}$. This is somewhat inconsistent with two-runner models whereby running behind can be an optimal strategy due in part to the drafting benefit ${ }^{27-28}$. These two-runner models ${ }^{27-28}$ however, involve faster speeds and correspondingly higher drafting benefit, and do not necessarily extend to larger numbers of runners where cumulative drafting benefit may be attained from more than one runner directly in front. This therefore suggests that this acceleration capacity at the end of a race results from lower levels of cognitive fatigue resulting from a reduced requirement to make continual decisions relating to muscular work rate ${ }^{20,21}$, at least in larger groups and at slightly lower speeds. It also suggest that the influence of the behavior of other competitors may be greater than the influence of afferent feedback on metabolic status in determining the work rate selected, at least in the early stages of a race. Towards the end, increasing metabolic disruption will cause slower runners to further reduce their speed, thereby resulting in incomplete realisation of performance potential ${ }^{8}$.

Furthermore, protocooperative behavior theory suggests that groups will tend to sort such that the MSO range among group members is approximately equivalent to the percentage energy savings from drafting ${ }^{15}$. In this study, the MSO range among the runners was $6.73 \%$ (max MSO $-\min \mathrm{MSO} / \max \mathrm{MSO}$ ), which is within the expected percent energy savings from drafting. This might suggest, speculatively, that the group has "pre-sorted" through earlier competitions, and thus narrowed to an MSO range equivalent to the energy saved by drafting. This suggestion is 
consistent with the work of Hanley ${ }^{19}$ who demonstrated that in elite runners group sorting tends to occur among competitors within a narrow range of similar ability.

One limitation of our study is that we did not analyse positional change, which is a feature of protocooperative behavior that generally occurs at comparatively low outputs ${ }^{15}$. Since drafting attenuates metabolic cost, we would expect high frequency positional change where there is high drafting quantity. Even without drafting, when speeds fall sufficiently relative to mean runners' MSO, we would expect some positional change as runners compete for desired tactical positions. Conversely, at high relative speeds, we would expect runners to reduce the number and frequency of positional changes within the group. Future studies may involve more specific analysis of durations for which certain positions are maintained. Again, analysis of sub-group formations were not undertaken here, and future studies may involve analysis of the mean MSO of sub-groups that form during the race. It should also be acknowledged that runners may have deliberately adopted specific intermediate positions due to perceived tactical benefits. However, detailed analysis of the effects of tactical positioning on finishing position is beyond the scope of this study.

The finding that the effects of (realistic) drafting on collective behavior is negligible would be expected to be especially relevant amongst groups of competitors of a lower performance level (or who compete in longer events) than were studied in this analysis. This suggests that where there is virtually no drafting advantage, runners tend to sort into groups of even narrower ranges of ability (i.e. runners sort into groups whose members possess nearly identical MSO). A potential limitation of this study is that we used athlete's season's best performances as individuals MSOs. We acknowledge that these may not be truly representative of absolute performance capacity because of to the relative infrequency at which track events of this distance are contested, and the tactical nature of many of these races. Nevertheless, we consider using seasons best to be more appropriate than all time personal record for this purpose due to the potentially long periods of time between this race and the setting of the personal record.

Our results show there are differences between simulations comparing no drafting with an unrealistic cycling drafting quantity, but there are smaller benefits realised at a more realistic running drafting quantity. If there were a greater benefit from drafting, the competitive MSO range might be greater, and so the results are not inconsistent with protocooperative theory. Also, since realistic drafting does not influence collective dynamics, collective dynamics would appear to be determined by mechanisms other than potential or perceived energetic savings.

Conclusion: Simulations indicate that the comparatively low drafting benefit obtained by runners does not have substantial effects on collective behavior. We would expect to see substantial differences in collective behavior only if the drafting benefit is considerably higher, likely somewhere between the realistic drafting quantity (up to $\sim 8 \%$ for runners) and the drafting quantity that cyclists experience. This finding indicates that group pacing behaviors in runners are not dominated by drafting, and that other (probably psychological) factors determine observed pacing behaviors. The results of our study are not inconsistent with protocooperative behavior theory which contends that group sorting tends to converge on the range of maximal abilities that is approximately equivalent to the energy saved by drafting. One implication of this 
is that where there is little or no drafting, groups will eventually sort so that groups contain runners of nearly identical potential performance capacity.

Practical applications: The key finding that collective behaviors in runners, at least from simulation models, cannot be explained through the energetic savings obtained by drafting has potentially important practical applications. It would suggest that athlete decision-making is influenced by behaviors displayed by other competitors and may well result in the selection of sub-optimal pacing strategies. Interventions designed to improve the quality of athlete decisionmaking may result in better utilisation of existing physiological resources and greater realisation of potential performance capacity.

Future research may involve video and/or more fine-grained speed data for positional and stretch dynamics, which may provide further insights into runners' collective dynamics, pacing strategies and general protocooperative behavior theory. This study involved analysis of performance data from a single elite championship $10,000 \mathrm{~m}$ race whereby reward is associated with position rather than the time achieved. It is not clear if similar results would be found in an analysis of female athletes, in a less homogenous sample of athletes, or in events of different durations. It is also not clear as to whether deliberate engagement in collective race behaviors that may maximise energetic savings from drafting, reduce cognitive load, or both, is likely to be any more or less effective in terms of maximising performance potential than would be selection of a more 'even paced' strategy that is typically considered optimal in events of this duration.

\section{References}

1. Foster C, Snyder AC, Thompson NN, Green MA, Foley M, Schrager M. Effect of pacing strategy on cycle time trial performance. Med Sci Sports Exerc. 1993; 383-388.

2. Tucker, R. The anticipatory regulation of performance: the physiological basis for pacing strategies and the development of a perception-based model for exercise performance. Br J Sports Med, 2009; 43(6), 392-400.

3. De Koning, J. J., Foster, C., Bakkum, A., Kloppenburg, S., Thiel, C., Joseph, T., Cohen, J. \& Porcari, J. P. Regulation of pacing strategy during athletic competition. PloS one, 2011; 6(1), e15863.

4. Baron, B., Moullan, F., Deruelle, F., \& Noakes, T. D. The role of emotions on pacing strategies and performance in middle and long duration sport events. Br J Sports Med, 2011; 45(6), 511-517.

5. Renfree, A., West, J., Corbett, M., Rhoden, C., \& St Clair Gibson, A. Complex interplay between determinants of pacing and performance during $20 \mathrm{~km}$ cycle time trials. Int J Sports Physiol Perf, 2012; 7(2), 121-129. 
6. Renfree, A., Martin, L., Micklewright, D., \& St Clair Gibson, A. Application of decision-making theory to the regulation of muscular work rate during self-paced competitive endurance activity. Sports Med, 2014; 44(2), 147-158.

7. Smits, B. L., Pepping, G. J., \& Hettinga, F. J. Pacing and decision making in sport and exercise: the roles of perception and action in the regulation of exercise intensity. Sports Med, 2014; 44(6), 763775 .

8. Renfree, A., \& St Clair Gibson, A. Influence of different performance levels on pacing strategy during the female World Championship marathon race. Int J Sports Physiol Perf, 2013; 8(3), 279-285.

9. Esteve-Lanao, J., Larumbe-Zabala, E., Dabab, A., AlcocerGamboa, A., \& Ahumada, F. Running World Cross Country Championships: A unique model for pacing study. Int J Sports Physiol Perf. 2014; 9(6), 1000-1005.

10. Hanley, B. Senior men's pacing profiles at the IAAF World Cross Country Championships. J Sports Sci. 2014;32(11):1060-1065.

11. Banerjee, A. V. A simple model of herd behavior. The Quarterly Journal of Economics, 1992; 197(3), 797-817.

12. Helbing, D., Buzna, L., Johansson, A., \& Werner, T. Selforganized pedestrian crowd dynamics: Experiments, simulations, and design solutions. Transportation Science, 2005; 39(1), 1-24.

13. Cont, R., \& Bouchaud, J. P. Herd behavior and aggregate fluctuations in financial markets. Macroeconomic Dynamics, 2000; 4(02), 170-196.

14. Wolfram, S. Complex systems theory. In Emerging Syntheses in Science: Proceedings of the Founding Workshops of the Santa Fe Institute (Addison-Wesley, 1988). 1985

15. Trenchard, H. The peloton superorganism and protocooperative behavior. Applied Mathematics and Computation, 2015; 270, 179192.

16. Kyle, C. Reduction of wind resistance and power output of racing cyclists and runners travelling in groups, Ergonomics, 1988; 22(4), 387-397 
17. Pugh, L.G. The influence of wind resistance in running and walking and the mechanical efficiency of work against horizontal or vertical forces. J Physiol, 1971; (213), 255-276.

18. Davies, C.T. Effects of wind assistance and resistance on the forward motion of a runner. $J$ Appl Physiol Respir Environ Exerc Physiol, 1980; 48(4), 702-9.

19. Hanley, B. Pacing profiles and pack running at the IAAF World Half Marathon Championships. J Sports Sci, 2015; 33(11), 11891195.

20. H. Zouhal, A. Ben Abderrahman, J. Proulx, B. Knechtle, L. Bougerra, W. Kebsi, T. Noakes. Drafting's improvement of 3000$\mathrm{m}$ running performance in elite athletes: is it a placebo effect? Int $J$ Sports Physiol Perf, 2015; 10(2), 147-152.

21. Renfree, A., Crivoi do Carmo, E., Martin, L., \& Peters, D. M. The influence of collective behaviour on pacing in endurance competitions. Frontiers Physiol, 2015; 6, 373.

22. Trenchard, H., Ratamero, E., Richardson, A., Perc, M. A deceleration model for bicycle peloton dynamics and group sorting. Applied Mathematics and Computation, 2015; 251, 24-34.

23. Ratamero, E. Modelling peloton dynamics in competitive cycling: a quantitative approach, Revised selected papers, Sports Science Research and Technical Support, International Congress ICSports 2015, eds J. Cabri, P. Pezarat Correia, J. Barreiros, Springer Intl, Switzerland

24. Wilensky, U. NetLogo. http://ccl.northwestern.edu/netlogo/. Center for Connected Learning and Computer-Based Modeling, Northwestern University, Evanston, IL, 1999.

25. Olds, T. The mathematics of breaking away and chasing in cycling, Eur J Appl Physiol, 1998; 77(6), 492-497.

26. McCole, S., Claney, K., Conte, J.C., Anderson, R., Hagberg, J. Energy expenditure during bicycling, J Appl Physiol, 1990; 68(2), 748-753.

27. Sullivan, G.M., Feinn, R. Using effect size - or why the $P$ value is not enough, J Grad Med Educ, 2012; 4(3), 279-282. 
537

538

539

540

541

542

543

544

545

546

547

548

549

550

551

552

553

554

555

556

557

558

559

560

561

562

563

564

565

566

567

568

569

570

571

572

573

574

575

576

577

578

579

580

581

582
28. Aftalion, A., Fiorini, C. A two-runners model: optimization of running strategies according to the physiological parameters, arXiv preprint, 2015; arXiv: 1508.00523

29. Pitcher, A. Optimal strategies for a two-runner model of middledistance running, SIAM J Appl Math, 2009; 70(4), 748-753. 


\section{Captions for Figures}

584

585

586

587

588

589

590

591

592

593

594

595

596

597

598

599

600

601

602

603

Figure 1: Individual competitors' speeds with moving average of winner.

Figure 2: Point in time from typical simulation trial showing individual maximal sustainable speeds converted from each runner's season best 10,000 m times; group stretch is distance (m) from first to last runner.

Figure 3: Mean speeds in simulated races in three different drafting conditions $\left({ }^{*} P<0.01\right)$.

Figure 4: Runner Convergence Ratio in simulated races in three different drafting conditions $(* P<0.01)$.

Figure 5: Mean stretch at each $100 \mathrm{~m}$ point in three different drafting conditions 


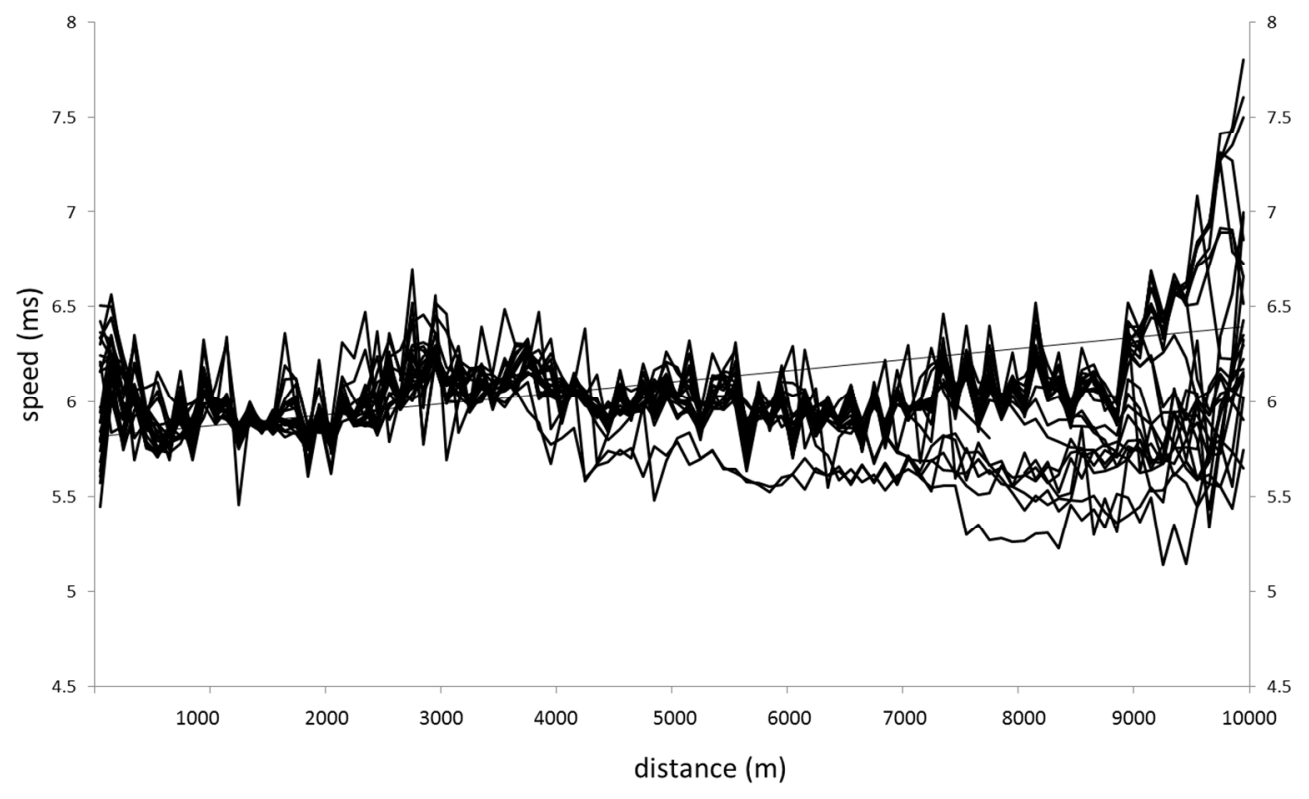

Figure 1: Individual competitors' speeds with moving average of winner. $355 \times 215 \mathrm{~mm}(300 \times 300 \mathrm{DPI})$ 


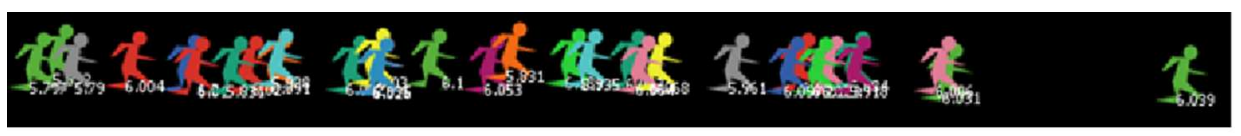

Figure 2: Point in time from typical simulation trial showing individual maximal sustainable speeds converted from each runner's season best 10,000 m times; group stretch is distance $(\mathrm{m})$ from first to last runner. $262 \times 109 \mathrm{~mm}(96 \times 96 \mathrm{DPI})$ 


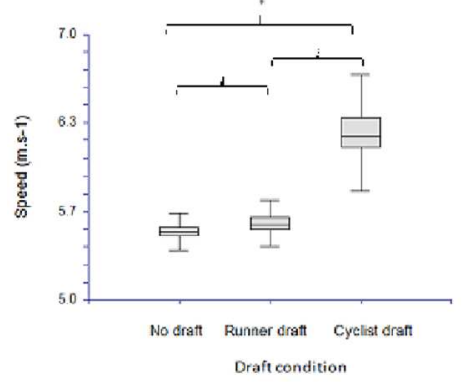

Figure 3: Mean speeds in simulated races in three different drafting conditions ( $\left.{ }^{*} P<0.01\right)$. $359 \times 152 \mathrm{~mm}(96 \times 96 \mathrm{DPI})$ 


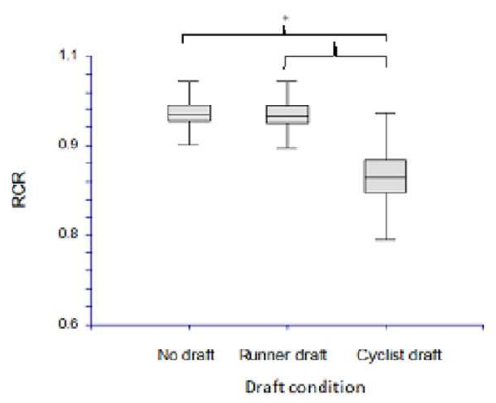

Figure 4: Runner Convergence Ratio in simulated races in three different drafting conditions $(* P<0.01)$. $361 \times 203 \mathrm{~mm}(96 \times 96 \mathrm{DPI})$ 


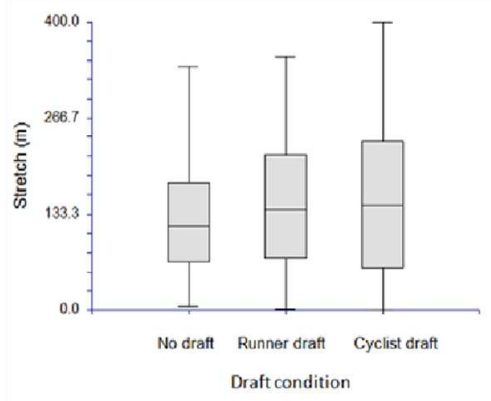

Figure 5: Mean stretch at each $100 \mathrm{~m}$ point in three different drafting conditions $359 \times 152 \mathrm{~mm}(96 \times 96 \mathrm{DPI})$ 